\title{
GENERALIZATION OF AN INEQUALITY OF ALZER FOR NEGATIVE POWERS
}

\author{
CHAO-PING CHEN AND FENG QI
}

\begin{abstract}
Let $\left\{a_{n}\right\}_{n=1}^{\infty}$ be a positive, strictly increasing, and logarithmically concave sequence satisfying $\left(a_{n+1} / a_{n}\right)^{n}<\left(a_{n+2} / a_{n+1}\right)^{n+1}$. Then we have

$$
\frac{a_{n}}{a_{n+m}}<\left(\frac{1}{n} \sum_{i=1}^{n} a_{i}^{r} / \frac{1}{n+m} \sum_{i=1}^{n+m} a_{i}^{r}\right)^{1 / r},
$$

where $n, m$ are natural numbers and $r$ is a positive real number. The lower bound is the best possible. This generalizes an inequality of Alzer for negative powers.
\end{abstract}

\section{Introduction}

When studying a problem on upper bound for permanents of $(0,1)$-matrices, in 1964 H. Minc and L. Sathre [5] discovered several noteworthy inequalities involving $(n !)^{1 / n}$. One of them is the following: If $n$ is a positive integer, then

$$
\frac{n}{n+1}<\frac{\sqrt[n]{n !}}{\sqrt[n+1]{(n+1) !}}<1
$$

By investigating a problem on Lorentz sequence spaces, in $1988 \mathrm{~J}$. S. Martins [4] published another lower bound for $\sqrt[n]{n !} / \sqrt[n+1]{(n+1) !}$ : Let $r$ be a positive real number and let $n$ be a natural number, then

$$
\left(\frac{1}{n} \sum_{i=1}^{n} i^{r} / \frac{1}{n+1} \sum_{i=1}^{n+1} i^{r}\right)^{1 / r} \leq \frac{\sqrt[n]{n !}}{\sqrt[n+1]{(n+1) !}} .
$$

Received February 10, 2004; revised April 15, 2004.

2000 Mathematics Subject Classification. Primary 26D15.

Key words and phrases. Alzer's inequality, logarithmically concave sequence, mathematical induction.

The authors were supported in part by NSF (\#10001016) of China, SF for the Prominent Youth of Henan Province (\#0112000200), SF of Henan Innovation Talents at Universities, Doctor Fund of Jiaozuo Institute of Technology, CHINA. 
In 1993 H. Alzer [1] compared the lower bounds of (1) and (2), and established the following result: Let $n$ be a positive integer, then for any positive real numbe $r$,

$$
\frac{n}{n+1} \leq\left(\frac{1}{n} \sum_{i=1}^{n} i^{r} / \frac{1}{n+1} \sum_{i=1}^{n+1} i^{r}\right)^{1 / r}
$$

The proof given by Alzer is remarkable, but it is quite long and complicated. Several easy proofs of (3) have been published by different authors, see $[2,7,8]$, and these proofs show that in fact (3) holds with strictly inequality. By mathematical induction and Cauchy's mean-value theorem, F. Qi [6] generalized the inequality (3) and showed that: Let $n$ and $m$ be natural numbers, $k$ a nonnegative integer, then

$$
\frac{n+k}{n+m+k}<\left(\frac{1}{n} \sum_{i=k+1}^{n+k} i^{r} / \frac{1}{n+m} \sum_{i=k+1}^{n+m+k} i^{r}\right)^{1 / r}
$$

where $r$ is any given positive real number. The lower bound is the best possible. In fact, (4) is essentially equivalent to

$$
\frac{n}{n+m}<\left(\frac{1}{n} \sum_{i=1}^{n} i^{r} / \frac{1}{n+m} \sum_{i=1}^{n+m} i^{r}\right)^{1 / r} .
$$

In this paper, the inequalities (3) and (5) are further generalized as follows.

Theorem. Let $\left\{a_{n}\right\}_{n=1}^{\infty}$ be a positive and strictly increasing sequence satisfying

$$
\frac{a_{n}}{a_{n+1}} \leq \frac{a_{n+1}}{a_{n+2}}, \quad n \in \mathbb{N}:=\{1,2, \ldots\}
$$

and

$$
\left(\frac{a_{n+1}}{a_{n}}\right)^{n}<\left(\frac{a_{n+2}}{a_{n+1}}\right)^{n+1}, \quad n \in \mathbb{N}
$$

Then we have

$$
\frac{a_{n}}{a_{n+m}}<\left(\frac{1}{n} \sum_{i=1}^{n} a_{i}^{r} / \frac{1}{n+m} \sum_{i=1}^{n+m} a_{i}^{r}\right)^{1 / r}
$$

where $n, m \in \mathbb{N}$ and $r$ is a positive real number. The lower bound is the best possible.

Notice that if a positive sequence $\left\{a_{n}\right\}_{n=1}^{\infty}$ satisfies the inequality (6), then we call it a logarithmically concave sequence.

Proof. The inequality (8) can be written as

$$
\frac{1}{(n+m) a_{n+m}^{r}} \sum_{i=1}^{n+m} a_{i}^{r}<\frac{1}{n a_{n}^{r}} \sum_{i=1}^{n} a_{i}^{r},
$$


which is equivalent to

$$
\frac{1}{(n+1) a_{n+1}^{r}} \sum_{i=1}^{n+1} a_{i}^{r}<\frac{1}{n a_{n}^{r}} \sum_{i=1}^{n} a_{i}^{r} .
$$

Since

$$
\sum_{i=1}^{n+1} a_{i}^{r}=\sum_{i=1}^{n} a_{i}^{r}+a_{n+1}^{r},
$$

(9) reduces to

$$
\sum_{i=1}^{n} a_{i}^{r}>\frac{n a_{n}^{r} a_{n+1}^{r}}{(n+1) a_{n+1}^{r}-n a_{n}^{r}} .
$$

It is easy to see that the inequality (10) holds for $n=1$. Suppose that the inequality (10) holds for some $n=k(k \geq 1)$, that is

$$
\sum_{i=1}^{k} a_{i}^{r}>\frac{k a_{k}^{r} a_{k+1}^{r}}{(k+1) a_{k+1}^{r}-k a_{k}^{r}} .
$$

Adding $a_{k+1}^{r}$ to the both sides of (11), we have

$$
\sum_{i=1}^{k+1} a_{i}^{r}>\frac{(k+1) a_{k+1}^{2 r}}{(k+1) a_{k+1}^{r}-k a_{k}^{r}} .
$$

By mathematical induction, it remains to show that

$$
\sum_{i=1}^{k+1} a_{i}^{r}>\frac{(k+1) a_{k+1}^{r} a_{k+2}^{r}}{(k+2) a_{k+2}^{r}-(k+1) a_{k+1}^{r}} .
$$

From (12) and (13) it is sufficient to show that

$$
\frac{(k+1) a_{k+1}^{2 r}}{(k+1) a_{k+1}^{r}-k a_{k}^{r}}>\frac{(k+1) a_{k+1}^{r} a_{k+2}^{r}}{(k+2) a_{k+2}^{r}-(k+1) a_{k+1}^{r}},
$$

which can be rearranged as

$$
(k+1)\left(\frac{a_{k+1}}{a_{k+2}}\right)^{r}-k\left(\frac{a_{k}}{a_{k+1}}\right)^{r}<1 .
$$

We difine for $r>0$

$$
f(r)=(k+1)\left(\frac{a_{k+1}}{a_{k+2}}\right)^{r}-k\left(\frac{a_{k}}{a_{k+1}}\right)^{r} .
$$

Differentiation yields

$$
\begin{aligned}
f^{\prime}(r) & =(k+1)\left(\frac{a_{k+1}}{a_{k+2}}\right)^{r} \ln \left(\frac{a_{k+1}}{a_{k+2}}\right)-k\left(\frac{a_{k}}{a_{k+1}}\right)^{r} \ln \left(\frac{a_{k}}{a_{k+1}}\right) \\
& =-\left(\frac{a_{k+1}}{a_{k+2}}\right)^{r} \ln \left(\frac{a_{k+2}}{a_{k+1}}\right)^{k+1}+\left(\frac{a_{k}}{a_{k+1}}\right)^{r} \ln \left(\frac{a_{k+1}}{a_{k}}\right)^{k} .
\end{aligned}
$$


Since

It is easy to see that

$$
\begin{gathered}
0<\frac{a_{k}}{a_{k+1}} \leqslant \frac{a_{k+1}}{a_{k+2}}, \quad k \in \mathbb{N} \\
1<\left(\frac{a_{k+1}}{a_{k}}\right)^{k}<\left(\frac{a_{k+2}}{a_{k+1}}\right)^{k+1}, \quad k \in \mathbb{N} .
\end{gathered}
$$

$$
\left(\frac{a_{k}}{a_{k+1}}\right)^{r} \ln \left(\frac{a_{k+1}}{a_{k}}\right)^{k}<\left(\frac{a_{k+1}}{a_{k+2}}\right)^{r} \ln \left(\frac{a_{k+2}}{a_{k+1}}\right)^{k+1}
$$

which implies that $f^{\prime}(r)<0$ and $f(r)<f(0)=1$, and then (14) holds.

By L' Hospital rule, easy caculation produces

$$
\lim _{r \rightarrow+\infty}\left(\frac{1}{n} \sum_{i=1}^{n} a_{i}^{r} / \frac{1}{n+m} \sum_{i=1}^{n+m} a_{i}^{r}\right)^{1 / r}=\frac{a_{n}}{a_{n+m}},
$$

thus, the lower bound given in (8) is the best possible. The proof is complete.

The authors [3] showed that (3) holds strictly for all natural numbers $n$ and all real numbers $r$. Now we pose the following open problem.

Open Problem. What conditions does the sequence $\left\{a_{k}\right\}_{k=1}^{\infty}$ satisfy such that (8) holds for all natural numbers $n, m$ and all real numbers $r$ ?

\section{References}

[1] H. Alzer, On an inequality of H. Minc and L. Sathre, J. Math. Anal. Appl. 179 (1993), 396-402.

[2] Ch.-P. Chen and F. Qi, Notes on proofs of Alzer's inequality, Octogon Mathematical Magazine 11 (2003), 29-33.

[3] Ch.-P. Chen and F. Qi, Extension of H. Alzer's inequality for negative powers, Tamkang Journal of Mathematics 36 (2005), 69-72.

[4] J. S. Martins, Arithmetic and geometric means, an application to Lorentz sequence spaces, Math. Nachr. 139 (1988), 281-288.

[5] H. Minc and L. Sathre, Some inequalities involving $(r !)^{1 / r}$, Proc. Edinburgh Math. Soc. $14(1964 / 65), 41-46$.

[6] F. Qi, Generalization of H. Alzer's inequality, J. Math. Anal. Appl. 240 (1999), 294-297.

[7] J. Sándor, On an inequality of Alzer, J. Math. Anal. Appl. 192 (1995), 1034-1035.

[8] J. S. Ume, An elementary proof of H. Alzer's inequality, Math. Japon. 44 (1996), 521-522.

Department of Applied Mathematics and Informatics, Henan Polytechnic University, Jiaozuo City, Henan 454010, CHINA.

E-mail: chenchaoping@sohu.com

Department of Applied Mathematics and Informatics, Henan Polytechnic University, Jiaozuo City, Henan 454010, CHINA.

E-mail: qifeng@jzit.edu.cn 